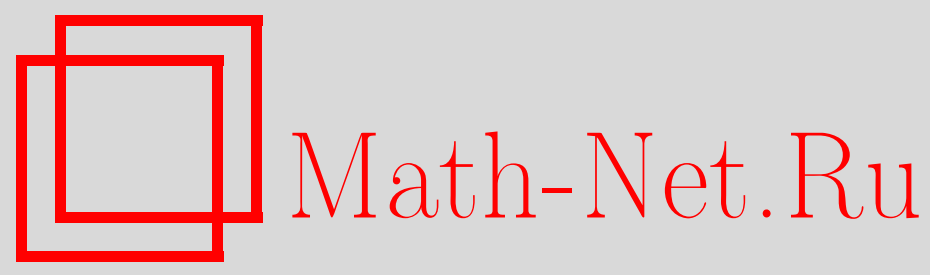

И. Д. Кан, Проблема Фробениуса для классов полиномиальной разрешимости, Матем. заметки, 2001, том 70, выпуск $6,845-853$

DOI: https://doi.org/10.4213/mzm797

Использование Общероссийского математического портала Math-Net.Ru подразумевает, что вы прочитали и согласны с пользовательским соглашением http://www . mathnet.ru/rus/agreement

Параметры загрузки:

IP: 54.205 .225 .156

26 апреля 2023 г., 13:02:12

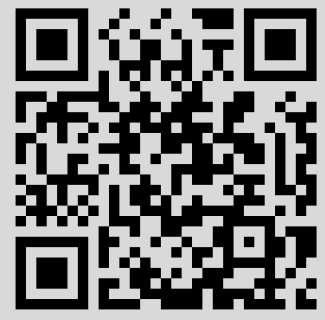




\section{ПРОБЛЕМА ФРОБЕНИУСА}

\section{ДЛЯ КЛАССОВ ПОЛИНОМИАЛЬНОЙ РАЗРЕШИМОСТИ}

\section{И. Д. Кан}

Проблема Фробениуса состоит в нахождении способа (= алгоритма) вычисления наибольшей "суммы денег", которую нельзя выдать монетами, имеющими взаимно простые достоинства $b_{0}, b_{1}, \ldots, b_{w}$. В качестве приемлемых (алгоритмов) решений принято рассматривать полиномиальные, названные так по форме зависимости затрат времени от длины исходной информации. О трудности проблемы Фробениуса говорит тот факт, что вопрос о сушествовании полиномиального решения уже для $w=3$ остается открытьм. В настоящей статье выделяются некоторые классы аргументов, на которых проблема решается полиномиально; между тем, рассуждения в духе теории сложности алгоритмов сведены к минимуму.

Библиография: 9 названий.

Настоящая работа посвящается моему учителю профессору Н. М. Коробову в знак глубокой признательности и по случаю его дня рождения.

1. Введение. Для всякой конечной последовательности натуральных чисел $B=$ $\left\{b_{0}, b_{1}, \ldots, b_{w}\right\}(w \geqslant 1)$ через $\langle B\rangle$ обозначим множество значений линейной формы

$$
\chi_{B}\left(u_{0}, u_{1}, \ldots, u_{w}\right)=b_{0} u_{0}+b_{1} u_{1}+\cdots+b_{w} u_{w}
$$

от целых неотрицательных переменных $u_{0}, u_{1}, \ldots, u_{w}$. Если элементы последовательности $B$ взаимно просты (в совокупности), то проблема Фробениуса состоит в нахождении наибольшего целого числа $g(B)$, не принадлежащего множеству $\langle B\rangle$. Таким образом,

$$
g(B)=\max (\mathbb{Z} \backslash\langle B\rangle) .
$$

Как правило, проблему Фробениуса рассматривают на бесконечных классах $\mathfrak{B}$ последовательностей $B$. Говоря упрощенно, алгоритм решения проблемы назьвается $n o$ линомиальным на классе $\mathfrak{B}$, если найдется полином $P(t)$ такой, что для любого набора $B \in \mathfrak{B}$ входных параметров $b_{0}, b_{1}, \ldots, b_{w}$ время работы этого алгоритма не превосходит величины $P\left(\sum_{i=0}^{w}\left(\log _{2} b_{i}+1\right)\right)$. Полиномиальные алгоритмы считаются достаточно быстрыми, т.е. приемлемьми для использования. Хотя надежда на полиномиальную разрешимость проблемы Фробениуса в целом вряд ли оправдана (Шевченко [1], там же-

Работа выполнена при частичной финансовой поддержке Российского фонда фундаментальных исследований, грант № 96-01-00492. 
точное определение полиномиальности алгоритма), для отдельных классов последовательностей такие решения известны: например, алгоритм Джонсона [2] для $w=2$ или алгоритм Рёдсета [3] для случая, когда $B \backslash\left\{b_{w}\right\}$ - арифметическая прогрессия.

В [4] проблема $\Phi$ робениуса решена полиномиально на классе иепны $x$ последовательностей $D=\left\{d_{0}, d_{1}, \ldots, d_{k}\right\}(k \geqslant 1)$, т.е. таких, что все отношения

$$
r_{D}(j)=\frac{d_{j-1}+d_{j+1}}{d_{j}}, \quad j=1,2, \ldots, k-1
$$

целые. Важную роль в исследовании множества $\langle D\rangle$ играют следующие объекты:

1) цепная дробь

$$
\varepsilon_{D}=1 /\left(r_{D}(1)-1 /\left(r_{D}(2)-\cdots-1 / r_{D}(k-1)\right) \ldots\right)
$$

со знаменателем $q_{D} \in \mathbb{N}$ своего несократимого представления;

2) число $\rho_{D}=d_{1}-\varepsilon_{D} d_{0}$ со свойством (см. [4, лемма 2])

$$
\rho_{D}=\frac{d_{k}}{q_{D}}>0
$$

3) функция

$$
x_{D}(y)=d_{1} y-d_{0}\left[\varepsilon_{D} y\right]=\rho_{D} y+d_{0}\left\{\varepsilon_{D} y\right\}
$$

аргумента $y \in \mathbb{Z}$, где для $\alpha \in \mathbb{R}$ используются обозначения

$$
[\alpha]=\max \{a \in \mathbb{Z} \mid a \leqslant \alpha\}, \quad\{\alpha\}=\alpha-[\alpha]
$$

- целая и дробная части.

Считаем, что при $k=1$ по определению $\varepsilon_{D}=0, q_{D}=1$.

Пусть множество $B$ представимо в виде

$$
B=\left\{b_{0}\right\} \cup A \cup C,
$$

где $A$ и $C$ - цепные последовательности, $A=\left\{a_{0}, a_{1}, \ldots, a_{m}\right\}, C=\left\{c_{0}, c_{1}, \ldots, c_{n}\right\}$, удовлетворяющие условиям

$$
a_{0} \equiv c_{0} \equiv 0\left(\bmod b_{0}\right), \quad m \geqslant 1, \quad n \geqslant 1, \quad \text { НОД }\left(b_{0}, a_{1}, c_{1}\right)=1 .
$$

Тогда последовательность $B$ назовем почти иепной.

Можно показать, что проблема Фробениуса на классе почти иепных последовательностей не проще, чем для произвольных пяти аргументов. Рассуждение состоит в превращении пятиэлементной последовательности в почти цепную путем добавления к ней некоторого количества фиктивных (зависимых) аргументов.

Цель настоящей работы - вычислить или оценить величину $g(B)$ при выполнении условий (1), (2). 
2. Основные обозначения. Определим числа $P_{-1}$ и $P_{0}, s_{-1}$ и $s_{0}$ условиями

$$
\begin{gathered}
P_{-1}=0, \quad P_{0}=\operatorname{HОД}\left(b_{0}, a_{1}\right), \\
s_{-1}=\frac{b_{0}}{P_{0}}, \quad \frac{s_{0} a_{1}}{P_{0}} \equiv c_{1}\left(\bmod s_{-1}\right), \quad 0 \leqslant s_{0}<s_{-1} .
\end{gathered}
$$

Рассматривая (3) как начальные условия, далее цепные последовательности $\left\{P_{j}\right\}$ и $\left\{s_{j}\right\}$ определим алгоритмом Евклида в форме Рёдсета [3], положив

$$
s_{j+1}=-\left[-\frac{s_{j-1}}{s_{j}}\right] s_{j}-s_{j-1}, \quad P_{j+1}=-\left[-\frac{s_{j-1}}{s_{j}}\right] P_{j}-P_{j-1}
$$

для $j=0,1, \ldots$, пока $s_{j} \neq 0$. Монотонность последовательностей $\left\{P_{j}\right\}$ и $\left\{s_{j}\right\}$, как и в [3], доказьвает убывание по $j$ их линейной комбинации

$$
R_{j}=\rho_{A} s_{j}-\rho_{C} P_{j}
$$

и единственность индекса $v$, корректно определенного условием

$$
R_{v+1} \leqslant 0<R_{v}
$$

Вычисление $s_{v}, s_{v+1}, P_{v}$ и $P_{v+1}$ можно сделать полиномиальным, аналогично теореме 3 из [5], что дает полиномиальность вычисления величины

$$
g_{2}=\max \left(x_{A}\left(s_{v}-s_{v+1}-1\right)+x_{C}\left(P_{v+1}-1\right), x_{A}\left(s_{v}-1\right)+x_{C}\left(P_{v+1}-P_{v}-1\right)\right)-b_{0} .
$$

Пусть $V_{G}$ - множество вершин многоугольника $G \subseteq \mathbb{R}^{2}$, определенного системой неравенств

$$
\begin{gathered}
0 \leqslant \lambda_{1} \leqslant 1, \quad 0 \leqslant \lambda_{2} \leqslant 1, \\
\lambda_{1} a_{0}\left\{-\varepsilon_{A} s_{v}\right\}+\lambda_{2} c_{0}\left\{\varepsilon_{C} P_{v}\right\}<R_{v}, \\
\lambda_{1} a_{0}\left\{\varepsilon_{A} s_{v+1}\right\}+\lambda_{2} c_{0}\left\{-\varepsilon_{C} P_{v+1}\right\} \leqslant-R_{v+1}
\end{gathered}
$$

в переменных $\lambda_{1}, \lambda_{2} \in \mathbb{R}$. Тогда $(0,0) \in G$ ввиду (5) и $\left|V_{G}\right| \leqslant 6$, где $|K|$ - количество элементов конечного множества $K$.

Для всякого непустого $\Pi \subseteq\left(G \cup V_{G}\right)$ положим

$$
\mathscr{H}(\Pi)=b_{0} \min _{\left(\lambda_{1}, \lambda_{2}\right) \in \Pi}\left[\frac{\left(1-\lambda_{1}\right) a_{0}\left(1-1 / q_{A}\right)+\left(1-\lambda_{2}\right) c_{0}\left(1-1 / q_{C}\right)}{b_{0}}\right] .
$$

Тогда, как известно из линейного программирования, $\mathscr{H}(G)=\mathscr{H}\left(V_{G}\right)$.

\section{3. Основные результаты.}

ТЕОРема 1. На классе почти чепных последовательностей В существует полиномиальный алгоритм вычисления величины $g_{1}=g_{1}(B)$ такой, что

$$
0 \leqslant g_{1}-g(B) \leqslant \mathscr{H}\left(V_{G}\right)<a_{0}+c_{0}
$$

в частности, если выполнены условия

$$
a_{0}=\min \left\{a_{0}, a_{1}, \ldots, a_{m-1}\right\}, \quad c_{0}=\min \left\{c_{0}, c_{1}, \ldots, c_{n-1}\right\},
$$

то оиенка (7) имеет место при $g_{1}=g_{2}$. 
ТЕОРема 2. Каждое (по отдельности) из условий

$$
\begin{gathered}
\min \left(R_{v},-R_{v+1}\right)>a_{0}+c_{0}-b_{0}-\frac{a_{0}}{q_{A}}-\frac{c_{0}}{q_{C}} \\
\min \left(m c_{0}, n a_{0}\right)=b_{0}
\end{gathered}
$$

является достаточным для выполнения равенства $\mathscr{H}\left(V_{G}\right)=0 u$, следовательно, выделяет в классе почти чепных последовательностей В подкласс, на котором величина $g(B)$ полиномиально вычислима.

ЗАМЕЧАНИЕ. Пусть вьполнены соотношения $b_{0}=a_{0}<a_{1}$ и $r_{A}(1)=r_{A}(2)=\cdots=$ $r_{A}(m-1)=2, n=1$. Тогда алгоритм вычисления величины $g(B)=g_{2}$ из теоремы 1 в целом совпадает с алгоритмом Рёдсета [3]. Исключение составляет выбор параметров (3), позволяющий избежать процедуры из [3], сводящей задачу к случаю НОД $\left(a_{0}, a_{1}\right)=1$.

4. Вспомогательные утверждения. В общем подходе к вычислению $g(B)$ используется формула Брауэра и Шокли [6]

$$
g(B)=\max \left\{t_{l} \mid l \in L\right\}-b_{0},
$$

где $L=\left\{0,1, \ldots, b_{0}-1\right\}$, а число $t_{l} \in\left\langle B \backslash\left\{b_{0}\right\}\right\rangle$ минимальное, сравнимое $c l$ по модулю $b_{0}$. В нашем случае ввиду (1)

$$
t_{l}=\min \left\{a+c \mid a \in\langle A\rangle, c \in\langle C\rangle, a+c \equiv l\left(\bmod b_{0}\right)\right\} .
$$

Следующее описание $\langle A\rangle$, по-существу, содержится в $[4$, леммы 1-5] (в других терминах).

ПРЕДЛОЖЕНИЕ 1. Принадлехсность $a \in\langle A\rangle$ равносильна существованию иельх неотричательных чисел и и у таких, что

$$
a=x_{A}(y)+u a_{0}
$$

Для любых $y, z \in \mathbb{Z}$ и $l \in L$ определим соответственно функцию $\sigma$ и множество $\Omega_{l}$, положив

$$
\begin{gathered}
\sigma(y, z)=x_{A}(y)+x_{C}(z) \\
\Omega_{l}=\left\{(y, z) \in \mathbb{Z}^{2} \mid y \geqslant 0, z \geqslant 0, \sigma(y, z) \equiv l\left(\bmod b_{0}\right)\right\} .
\end{gathered}
$$

Тогда из определения $x_{D}(D=A, C)$ и сравнений в (2) следует, что сравнение в (14) равносильно сравнению

$$
a_{1} y+c_{1} z \equiv l\left(\bmod b_{0}\right) .
$$

Лемма 1. Имеет место равенство

$$
g(B)=\max _{l \in L}\left(\min \left\{\sigma(y, z) \mid(y, z) \in \Omega_{l}\right\}\right)-b_{0} .
$$


ДокАЗАТЕЛЬСТво. Пусть $l \in L$ и $t_{l}=a+c$, как в (12). Тогда ввиду сравнений в (2) и минимальности $t_{l}$ имеем $a-a_{0} \notin\langle A\rangle, a \in\langle A\rangle$. Отсюда ввиду предложения 1 $a=x_{A}(y), y \geqslant 0$. Аналогично, $c=x_{C}(z), z \geqslant 0$. Так что

$$
t_{l}=x_{A}(y)+x_{C}(z)=\sigma(y, z), \quad y \geqslant 0, \quad z \geqslant 0,
$$

и (12) перепишется в виде

$$
t_{l}=\min \left\{\sigma(y, z) \mid(y, z) \in \Omega_{l}\right\} .
$$

Ввиду формулы (11) лемма доказана.

Главная трудность мини-, максимизации функции $\sigma$ - ее “негладкость". Для сглаживания фиксируем произвольное $\bar{\lambda}=\left(\lambda_{1}, \lambda_{2}\right) \in G$ и выделим в $\sigma$ "гладкую" составляющую $\tau$, положив для любых $y, z \in \mathbb{Z}$

$$
\tau(y, z)=\rho_{A} y+\rho_{C} z+\lambda_{1} a_{0}\left\{\varepsilon_{A} y\right\}+\lambda_{2} c_{0}\left\{\varepsilon_{C} z\right\} .
$$

Тогда из определения $x_{D}(D=A, C)$ следует, что

$$
\sigma(y, z)=\tau(y, z)+\left(1-\lambda_{1}\right) a_{0}\left\{\varepsilon_{A} y\right\}+\left(1-\lambda_{2}\right) c_{0}\left\{\varepsilon_{C} z\right\} .
$$

Для каждого $l \in L$ определим пару $\left(y_{l}, z_{l}\right) \in \Omega_{l}$ (формально зависящую от выбора $\bar{\lambda}$ ). Именно, пусть пара $\left(y_{l}, z_{l}\right)$ та (или любая из тех), что обладает (-ют) минимальной второй компонентой $z=z_{l}$ среди всех тех пар $(y, z)$, на которых достигается минимум функции $\tau(y, z)$ по множеству $\Omega_{l}$.

ЛЕмма 2. Если выбранное $\bar{\lambda} \in G$ удовлетворяет условию $\mathscr{H}(\{\bar{\lambda}\})=\mathscr{H}\left(V_{G}\right)$, mo

$$
0 \leqslant \max \left\{\sigma\left(y_{l}, z_{l}\right) \mid l \in L\right\}-b_{0}-g(B) \leqslant \mathscr{H}\left(V_{G}\right) .
$$

ДокАЗАТЕЛЬСТво. Если для $\alpha, \beta \in \mathbb{R}$ число $\alpha+\beta$ целое, то $\alpha+\beta=-[-\alpha]+[\beta]$. В частности, для $(y, z) \in \Omega_{l}$ ввиду $(14)$ и $(16)$

$$
\sigma(y, z)=f(y, z, l)+F(y, z),
$$

где

$$
f(y, z, l)=l-b_{0}\left[\frac{l-\tau(y, z)}{b_{0}}\right], \quad F(y, z)=b_{0}\left[\frac{\left(1-\lambda_{1}\right) a_{0}\left\{\varepsilon_{A} y\right\}+\left(1-\lambda_{2}\right) c_{0}\left\{\varepsilon_{C} z\right\}}{b_{0}}\right] .
$$

Поскольку

TO

$$
0 \leqslant\left\{\varepsilon_{A} y\right\} \leqslant 1-\frac{1}{q_{A}}, \quad 0 \leqslant\left\{\varepsilon_{C} z\right\} \leqslant 1-\frac{1}{q_{C}}
$$

$$
0 \leqslant F(y, z) \leqslant \mathscr{H}(\{\bar{\lambda}\})=\mathscr{H}\left(V_{G}\right) .
$$

Поэтому из (18) имеем

$$
\begin{aligned}
\sigma\left(y_{l}, z_{l}\right) & \geqslant \min _{(y, z) \in \Omega_{l}} \sigma(y, z) \geqslant \min _{(y, z) \in \Omega_{l}} f(y, z, l) \\
& =f\left(y_{l}, z_{l}, l\right)=\sigma\left(y_{l}, z_{l}\right)-F\left(y_{l}, z_{l}\right) \geqslant \sigma\left(y_{l}, z_{l}\right)-\mathscr{H}\left(V_{G}\right) .
\end{aligned}
$$

Максимизируя формально по $l \in L$, получаем отсюда ввиду леммы 1 неравенство (17). Лемма доказана.

Для максимизации величин $\sigma\left(y_{l}, z_{l}\right)$ оценим сверху их аргументы, а снизу, предварительно, - некоторые приращения функции $\tau$, положив

$$
\psi=-\tau\left(-s_{v}, P_{v}\right), \quad \varphi=-\tau\left(s_{v+1},-P_{v+1}\right) .
$$

Тогда из (4) и (6) следует, что $\psi>0, \varphi \geqslant 0$. 
Лемма 3. Имеют место оиенки

$$
\begin{gathered}
\tau\left(y_{l}, z_{l}\right)-\tau\left(y_{l}-s_{v}, z_{l}+P_{v}\right) \geqslant \psi>0, \\
\tau\left(y_{l}, z_{l}\right)-\tau\left(y_{l}+s_{v+1}, z_{l}-P_{v+1}\right) \geqslant \varphi \geqslant 0, \\
\tau\left(y_{l}, z_{l}\right)-\tau\left(y_{l}-s_{v}+s_{v+1}, z_{l}+P_{v}-P_{v+1}\right) \geqslant \psi+\varphi>0 .
\end{gathered}
$$

ДокАЗАТЕЛЬСТВо. Поскольку для любых $\alpha, \beta \in \mathbb{R}$

$$
\{\alpha\}-\{\beta\} \geqslant-\{\beta-\alpha\},
$$

то ввиду определения $\tau$ для любых $y, z, Y, Z \in \mathbb{Z}$

$$
\tau(y, z)-\tau(Y, Z) \geqslant-\tau(Y-y, Z-z) .
$$

Отсюда вытекают неравенства (20)-(22). Лемма доказана.

ЛЕмма 4 (почти по Рёдсету [3]). Для любого $l \in L$ nара $\left(y_{l}, z_{l}\right)$ удовлетворяет каждому из неравенств

$$
y_{l}<s_{v}, \quad z_{l}<P_{v+1}
$$

и, кроме того, хотя бы одному из неравенств

$$
y_{l}<s_{v}-s_{v+1}, \quad z_{l}<P_{v+1}-P_{v}
$$

ДокАЗАТЕЛЬСтво. Сравнения

$$
c_{1} P_{j} \equiv a_{1} s_{j}\left(\bmod b_{0}\right), \quad j=-1,0,1, \ldots, v+1,
$$

доказьваемые индукцией по $j$ с базой (3), показывают ввиду (15), что при неотрицательности аргументов вычитаемого в каждой из разностей в (20)-(22) пара этих аргументов принадлежит $\Omega_{l}$. Поэтому, предположив невьполнение утверждений леммы, приходим в противоречие с леммой 3. Действительно, строгое вьполнение неравенств (20)-(22) теперь невозможно из-за минимальности $\tau\left(y_{l}, z_{l}\right)$, а вырождение нестрогого неравенства (21) в цепочку равенств - из-за минимальности $z_{l}$. Лемма доказана.

Чтобы показать далее полноту ограничений леммы 4 на пару $\left(y_{l}, z_{l}\right)$, ввиду чего последняя фактически не зависит от выбора $\bar{\lambda} \in G$, положим

$$
\begin{gathered}
A_{1}=\left\{(y, z) \in \mathbb{Z}^{2} \mid 0 \leqslant y<s_{v}-s_{v+1}, 0 \leqslant z<P_{v+1}\right\} \\
A_{2}=\left\{(y, z) \in \mathbb{Z}^{2} \mid 0 \leqslant y<s_{v}, 0 \leqslant z<P_{v+1}-P_{v}\right\} \\
T=\left\{\sigma\left(y_{l}, z_{l}\right) \mid l \in L\right\}, \quad S_{i}=\left\{\sigma(y, z) \mid(y, z) \in A_{i}\right\}, \quad i=1,2 .
\end{gathered}
$$

ЛЕмма 5. Каково бы ни было значение $\bar{\lambda} \in G$, имеют место оченка (17) $u$ равенство

$$
\max \left\{\sigma\left(y_{l}, z_{l}\right) \mid l \in L\right\}=\max \left(S_{1} \cup S_{2}\right)
$$


ДоказАТЕЛЬСтво. Ввиду леммы 2 достаточно доказать равенство $T=S_{1} \cup S_{2}$. Для этого, почти цитируя Рёдсета [3], заметим следующее. Равенство

$$
s_{j} P_{j+1}-s_{j+1} P_{j}=b_{0}, \quad j=-1,0,1, \ldots, v,
$$

проверяемое индукцией по $j$ с базой (3), влечет

$$
\left|A_{1} \cup A_{2}\right|=\left|A_{1}\right|+\left|A_{2} \backslash A_{1}\right|=\left(s_{v}-s_{v+1}\right) P_{v+1}+s_{v+1}\left(P_{v+1}-P_{v}\right)=b_{0} .
$$

Поскольку ввиду леммы $4 T \subseteq S_{1} \cup S_{2}$, то

$$
b_{0}=|T| \leqslant\left|S_{1} \cup S_{2}\right| \leqslant\left|A_{1} \cup A_{2}\right|=b_{0},
$$

так что $T=S_{1} \cup S_{2}$. Лемма доказана.

Через $\max A_{i}$ для $i=1,2$ обозначим пару $(y, z) \in A_{i}$, обе компоненты которой максимальны.

Лемма 6. Из условий (8) следует, что

$$
\max S_{i}=\sigma\left(\max A_{i}\right), \quad i=1,2 .
$$

ДокАЗАТЕЛЬСтво. Ввиду (13) достаточно доказать неубьвание $x_{A}(y)\left(x_{C}(y)\right.$ - по аналогии). Если $r_{A}(j) \geqslant 2$ для всех $j=1,2, \ldots, m-1$, то $\left[-\varepsilon_{A}\right]=[1 / m-1]$. Поскольку ввиду (8) $m=1$ или $a_{1} \geqslant a_{0}$, то ввиду неравенства (23)

$$
x_{A}(y+1)-x_{A}(y) \geqslant-x_{A}(-1)=a_{1}+a_{0}\left[\frac{1}{m}-1\right] \geqslant 0
$$

и неубывание $x_{A}(y)$ доказано.

Теперь все случаи равенства $r_{A}(j)=1$ исключим индукцией по $m$. Именно, пусть $A(j)=\left\{a_{0}^{(j)}, a_{1}^{(j)}, \ldots, a_{m-1}^{(j)}\right\}-$ результат вычеркивания из $A$ одного любого некрайнего элемента $a_{j}$ такого, что $r_{A}(j)=1$. Тогда $A(j)$ - цепная последовательность, причем

$$
a_{0}^{(j)}=a_{0}, \quad a_{m-1}^{(j)}=a_{m}, \quad \varepsilon_{A(j)}=\varepsilon_{A}-\left[\frac{1}{j}\right]
$$

откуда

$$
q_{A(j)}=q_{A}, \quad\left\{\varepsilon_{A(j)}\right\}=\left\{\varepsilon_{A}\right\}, \quad \rho_{A(j)}=\rho_{A} .
$$

Следовательно, функция $x_{A}(y)=x_{A(j)}(y)$ не убывает по предположению индукции. Лемма доказана. 
5. Доказательство теорем. Положим

$$
g_{1}=\max \left(S_{1} \cup S_{2}\right)-b_{0} .
$$

Тогда ввиду леммы 5 имеет место оценка (7). Величина $g_{1}$ полиномиально вычислима методами Ленстры [7] или Кай-Ман Цана [8]. При выполнении условий (8) для вычисления $g_{1}$ используется лемма 6 , ввиду которой $g_{1}=g_{2}$, что завершает доказательство теоремы 1.

При выполнении (9) из оценок (19) следует существование числа $\mu$ такого, что $(\mu, \mu)$ $\in G$ и $\mathscr{H}(\{\mu, \mu\})=0$. Далее, равенство (10) означает, что $m=c_{0} / b_{0}=1$ или $n=a_{0} / b_{0}$ $=1$. Взяв для определенности первьй случай, найдем, что

$$
\varepsilon_{A}=0, \quad(1,0) \in G, \quad \mathscr{H}(\{1,0\})=0 .
$$

Поэтому теорема 2 следует из теоремы 1 . Теоремы доказаны.

\section{6. Следствия.}

СлЕДСТВИЕ 1. Если натуральные числа а и $b<a, a+1 \geqslant(1-b)[-a / b]$, то имеет место равенство

$$
\begin{gathered}
g(a, a+1, a+2, a+b, a+2 b)=(a+b)\left[\frac{a-1}{b}\right]+a b-2 a-1 \\
-\min \left(-b+(a b+b)\left\{-\frac{a}{b}\right\}+a\left[\frac{a-1}{2 b}\right]+a\left[\frac{b\{(a-1) / b\}}{2}\right],\right. \\
\left.a\left[\frac{b+1}{2}\right]+a\left[\frac{a-1-b}{2 b}\right]\right) .
\end{gathered}
$$

СЛЕДСТВИЕ 2. Если натуральные числа а и $2 \leqslant b<a, 2 a+3 \geqslant(3-2 b)[-a / b]$, то имеет место равенство

$$
\begin{aligned}
& g(a, a+1,2 a+3, a+b)=(a+b)\left[\frac{a-1}{b}\right]+a b-2 a-1 \\
& \quad-\min \left(-b+(a b+b)\left\{-\frac{a}{b}\right\}+a\left[\frac{b\{(a-1) / b\}}{3}\right], a\left[\frac{b+2}{3}\right]\right) .
\end{aligned}
$$

СЛЕДСТВИЕ 3. Пусть выполнены условия (8), $n=1, a_{0} \equiv 0\left(\bmod c_{1}\right) u$ НОД$\left(c_{1}\right.$, $\left.a_{1}\right)=1$. Тогда

$$
g\left(\left\{c_{1}\right\} \cup A\right)=a_{1} c_{1}-a_{1}-c_{1}-a_{0}\left[\varepsilon_{A}\left(c_{1}-1\right)\right]
$$

Аналогичный следствию 3 результат с заменой $A$ на арифметическую прогрессию получен Селмером [9]. 
ДокАЗАтЕльство слЕДСтвий. Аргументные множества из следствий 1,2 очевидным образом представляются объединением $B$ двух цепных последовательностей с общим элементом $a_{0}=c_{0}=b_{0}=a$. Для следствий 1 и 2 выполняются соответственно условия (9) и (10) из теоремы 2 , ввиду чего $\mathscr{H}\left(V_{G}\right)=0$, а ввиду вьполнения условия (8) из теоремы $1 g(B)=g_{2}$. Дальнейшие вычисления не представляют сложности, поскольку $v \leqslant 0$. Следствия 1 и 2 доказаны.

Для доказательства следствия 3 положим $c_{0}=b_{0}=a_{0}$. Тогда, как и вьше, ввиду (8) и $(10) g(B)=g_{2}$. Вычисления показьвают, что

$$
P_{v+1}=\frac{a_{0}}{c_{1}}, \quad s_{v+1}=0, \quad s_{v}=c_{1}
$$

ввиду чего информация о величине $P_{v}$ уже не является необходимой: подстановка значений $(25)$ в определение $g_{2}$ дает формулу (24). Следствие 3 доказано.

\section{СПИСОК ЦИТИРОВАННОЙ ЛИТЕРАТУРЫ}

[1] Шевченко В.Н. Качественные вопросы целочисленного программирования. М.: Наука, 1995.

[2] Johnson S. M. A linear diophantine problem // Canad. J. Math. 1960. V. 12. № 2. P. 390-398.

[3] Rödset Ö. J. On a linear diophantine problem of Frobenius II // J. Reine Angew. Math. 1979. V. 307/308. P. 431-440.

[4] Кан И. Д. Представление чисел линейньми формами // Матем. заметки. 2000. Т. 68. № 2. C. $210-216$.

[5] Кан И. Д. К проблеме Фробениуса // Фундаментальная и прикладная математика. 1997. Т. 3. № 3. С. $821-835$.

[6] Brauer A., Shockly J.E. On a problem of Frobenius // J. Reine Angew. Math. 1962. V. 211. №3/4. P. 215-220.

[7] Lenstra H. W. Integer programming with a fixed number of variables // Math. of Oper. Res. 1983. V. 8. № 4. P. 538-548.

[8] Kai-Man Tsang. On a linear diophantine problem of Frobenius // Studia Scientarum Math. Hungarica. 1988. V. 23. P. 443-452.

[9] Selmer E. S. On the linear diophantine problem of Frobenius // J. Reine Angew. Math. 1977. V. 293/294. P. 1-17. 